\title{
PROXIMALITY AND EQUIDISTRIBUTION ON THE FURSTENBERG BOUNDARY
}

\author{
A. GORODNIK AND F. MAUCOURANT
}

\begin{abstract}
Let $G$ be a connected semisimple Lie group with finite center and without compact factors, $P$ a minimal parabolic subgroup of $G$, and $\Gamma$ a lattice in $G$. We prove that every $\Gamma$-orbits in the Furstenberg boundary $G / P$ is equidistributed for the averages over Riemannian balls. The proof is based on the proximality of the action of $\Gamma$ on $G / P$.
\end{abstract}

\section{INTRODUCTION}

Let $G$ be a connected semisimple Lie group with finite center and without compact factor, and $\Gamma$ a lattice in $G$, that is, a discrete subgroup of $G$ such that $\Gamma \backslash G$ has finite volume. In this article we investigate the distribution of orbits of $\Gamma$ acting on the Furstenberg boundary of $G$. Recall that the Furstenberg boundary can be identified with the factor space $G / P$, where $P$ is a minimal parabolic subgroup of $G$. It is known that every orbit of $\Gamma$ in $G / P$ is dense (see $\underline{\mathrm{Mo}}$ ). We show that orbits of $\Gamma$ are equidistributed with respect to the averages over Riemannian balls.

Since we study the action of a nonamenable group on a space without a finite invariant measure, our result lies outside the scope of the classical ergodic theory. The published results about distribution of dense orbits of nonamenable groups are limited to a few special examples. Arnold and Krylov showed in [AK that dense orbits of groups generated by two rotations acting on the 2-dimensional sphere are equidistributed. A similar problem was considered by Kazhdan in $\mathrm{Ka}$ where he studied the action of a group generated by two affine isometries on the plane $\mathbb{R}^{2}$. Distribution of dense orbits of a lattice in $\operatorname{SL}(2, \mathbb{R})$ acting on $\mathbb{R}^{2}$ was investigated by Ledrappier $[\mathrm{L}$ and Nogueira $\mathbb{N}$.

Let $X$ be the symmetric space of $G$ equipped with a right invariant Riemannian metric $d$. Note that $X$ can be identified with $L \backslash G$ for a maximal compact subgroup $L$ of $G$.

Fix $x, \tilde{x} \in X$ and denote by $K$ and $\tilde{K}$ the stabilizers of $x$ and $\tilde{x}$ respectively. Let $\nu$ and $\tilde{\nu}$ be the probability Haar measures on $K$ and $\tilde{K}$ and $m_{\tilde{x}}$ the harmonic measures at $\tilde{x}$ on $G / P$, that is, the unique $\tilde{K}$-invariant probability measure on $G / P$. For $S \subset G$

The first author is partially supported by NSF grant 0400631. 
and $T>0$, define

$$
\begin{aligned}
S_{T}(\tilde{x}) & =\{s \in S: d(x, \tilde{x} s)<T\} \\
S_{T} & =S_{T}(x) .
\end{aligned}
$$

Our main result is the following theorem.

Theorem 1. For every $f \in C(G / P), \tilde{x} \in X$, and $y \in G / P$,

$$
\lim _{T \rightarrow \infty} \frac{1}{\left|\Gamma_{T}(\tilde{x})\right|} \sum_{\gamma \in \Gamma_{T}(\tilde{x})} f(\gamma y)=\int_{G / P} f d m_{\tilde{x}},
$$

Moreover, the convergence is uniform for $y \in G / P$.

We remark that it was shown in [EM] (see also [DRS]) that

$$
\left|\Gamma_{T}(\tilde{x})\right| \sim_{T \rightarrow \infty} \frac{\operatorname{Vol}\left(G_{T}(\tilde{x})\right)}{\operatorname{Vol}(\Gamma \backslash G)}
$$

and the exact asymptotics of the volume $\operatorname{Vol}\left(G_{T}(\tilde{x})\right)=\operatorname{Vol}\left(G_{T}\right)$ as $T \rightarrow \infty$ was computed in $\mathrm{Kn}$.

The first result in the direction of Theorem 1 was established in [Ma], where the case of the real hyperbolic spaces was considered. A different proof of Theorem 1 is given in [GO. An advantage of the approach presented here is that it shows that the convergence is uniform. While the proof in GO uses equidistribution of solvable flows on $\Gamma \backslash G$, our proof is based on the strong proximality of the action of $G$ on $G / P$ (see Theorem 2 below). This result is of independent interest, and it might be useful for other applications.

Recall that an action of a group $H$ on a compact metric space $(Y, d)$ is called proximal if for every $u, v \in Y$ there exists a sequence $\left\{h_{n}\right\} \subset H$ such that $d\left(h_{n} u, h_{n} v\right) \rightarrow 0$ as $n \rightarrow \infty$. The fact that the action of $G$ on $G / P$ is proximal plays important role in the study of random walks on $G$ (see, for example, $[\mathrm{F}]$ ). It turns out that a typical sequence in $G$ acts on $G / P$ in proximal fashion.

Theorem 2 (Strong proximality). Let $\mathcal{O}$ be neighborhood of the diagonal in $G / P \times$ $G / P$ and $u, v \in G / P$. Then

$$
\lim _{T \rightarrow \infty} \frac{\operatorname{Vol}\left(\left\{g \in G_{T}(\tilde{x}):(g u, g v) \notin \mathcal{O}\right\}\right)}{\operatorname{Vol}\left(G_{T}(\tilde{x})\right)}=0
$$

and

$$
\lim _{T \rightarrow \infty} \frac{\left|\left\{\gamma \in \Gamma_{T}(\tilde{x}):(\gamma u, \gamma v) \notin \mathcal{O}\right\}\right|}{\left|\Gamma_{T}(\tilde{x})\right|}=0
$$

uniformly on $u, v$.

In the case of the real hyperbolic space, Theorem 2 was proved in Ma using geometric methods. 


\section{Proof of Theorem 2}

2.1. Cartan decomposition. Let $G=K_{0} \exp (\mathfrak{p})$ be the Cartan decomposition of $G$ and $A \subset \exp (\mathfrak{p})$ a split Cartan subgroup of $G$, that is, a maximal connected abelian $\operatorname{subgroup}$ in $\exp (\mathfrak{p})$. We fix a system of positive roots $\Sigma^{+}$on $\mathfrak{a}=\operatorname{Lie}(A)$, and let

$$
A^{+}=\left\{a \in A: \alpha(\log a) \geq 0 \text { for all } \alpha \in \Sigma^{+}\right\}
$$

denote the closed positive Weyl chamber in $A$. Then $G=K A^{+} K$, and a Haar measure on $G$ can be given by

$$
\int_{G} \psi(g) d g=\int_{K} \int_{A^{+}} \int_{K} \psi\left(k_{1} a k_{2}\right) \xi(\log a) d \nu\left(k_{1}\right) d a d \nu\left(k_{2}\right), \quad \psi \in C_{c}(G),
$$

where $d a$ denotes the Lebesgue measure on $A$,

$$
\xi(s)=\prod_{\alpha \in \Sigma^{+}} \sinh (\alpha(s))^{m_{\alpha}}, \quad s \in \mathfrak{a},
$$

and $m_{\alpha}$ denotes the dimension of the root space for the root $\alpha \in \Sigma^{+}$.

Let $\tilde{g} \in G$ be such that $x \tilde{g}=\tilde{x}$. Then $G=\tilde{g}^{-1} K A^{+} K, G_{T}(\tilde{x})=\tilde{g}^{-1} K A_{T}^{+} K$, and

$$
\int_{G} \psi(g) d g=\int_{K} \int_{A^{+}} \int_{K} \psi\left(\tilde{g}^{-1} k_{1} a k_{2}\right) \xi(\log a) d \nu\left(k_{1}\right) d a d \nu\left(k_{2}\right), \quad \psi \in C_{c}(G) .
$$

In particular, it follows that

$$
\operatorname{Vol}\left(G_{T}(\tilde{x})\right)=\operatorname{Vol}\left(G_{T}\right)=\int_{A_{T}^{+}} \xi(\log a) d a .
$$

\subsection{Reduction to maximal parabolics. Fix a system of simple roots}

$$
\Pi=\left\{\alpha_{1}, \ldots, \alpha_{r}\right\} \subset \Sigma^{+} .
$$

Here $r=\operatorname{dim} A$ is the $\mathbb{R}$-rank of $G$. It is well-known that the closed subgroups of $G$ that contain $P$ are in one-to-one correspondence with the subsets of $\Pi$ (see $[$, Sec. 1.2]). In particular, $P_{i}=P_{\left\{\alpha_{i}\right\}}, i=1, \ldots, r$, are the maximal parabolic subgroups of $G$ and

$$
P=\bigcap_{i=1}^{r} P_{i}
$$

We consider the projection maps

$$
\pi_{i}: G / P \times G / P \rightarrow G / P_{i} \times G / P_{i}, \quad i=1, \ldots, r .
$$

Let $\Delta$ and $\Delta_{i}$ denote the diagonals in $G / P \times G / P$ and $G / P_{i} \times G / P_{i}$ respectively. Then

$$
\Delta=\bigcap_{i=1}^{r} \pi_{i}^{-1}\left(\Delta_{i}\right)
$$


Since

$$
\prod_{i=1}^{r} \pi_{i}: G / P \times G / P \rightarrow \prod_{i=1}^{r} G / P_{i} \times G / P_{i}
$$

is a continuous injective map from a compact space to a Hausdorff space, it is a homeomorphism onto its image. It follows that for any neighborhood $\mathcal{O}$ of $\Delta$ in $G / P \times G / P$, there exist neighborhoods $\mathcal{O}_{i}$ of $\Delta_{i}$ in $G / P_{i} \times G / P_{i}$ such that

$$
\mathcal{O} \supset \bigcap_{i=1}^{r} \pi_{i}^{-1}\left(\mathcal{O}_{i}\right)
$$

Then for every $(u, v) \in G / P \times G / P$,

$$
\{g \in G: g \cdot(u, v) \notin \mathcal{O}\} \subset \bigcup_{i=1}^{r}\left\{g \in G: g \cdot \pi_{i}(u, v) \notin \mathcal{O}_{i}\right\} .
$$

This inclusion shows that it suffices to prove Theorem 2 under the assumption that $P$ is a maximal parabolic subgroup of $G$. We keep this assumption until the end of this section.

2.3. Dynamics on projective space. By a result from [T], there is an irreducible representation $G \rightarrow \mathrm{GL}(V)$ such that the highest weight space is one-dimensional, and the stabilizer of this space is $P$. We consider the induced action of $G$ on the projective space $\mathbb{P}(V)$, and let $w^{+} \in \mathbb{P}(V)$ be the direction of the highest weight space. The map $g \mapsto g w^{+}$defines an embedding of $G / P$ in $\mathbb{P}(V)$. Note that if $\lambda$ is the highest weight, the other weights of the representation are of the form $\lambda-\sum_{\alpha \in \Sigma^{+}} n_{\alpha} \alpha$ for integers $n_{\alpha} \geq 0$. We denote by $V^{<}$the sum of all root spaces with weights other than $\lambda$. We fix a $K$-invariant scalar product on $V$, which gives rise to a metric $d$ on $\mathbb{P}(V)$, which is $K$-invariant. Put $\tilde{d}\left(w_{1}, w_{2}\right)=d\left(\tilde{g} w_{1}, \tilde{g} w_{2}\right)$. Let $V_{\varepsilon}^{<}$be the open $\varepsilon$-neighborhood of $V^{<}$in $\mathbb{P}(V)$ with respect to the metric $\tilde{d}$.

For $w \in \mathbb{P}(V)$ and $\tau>0$, define

$$
K_{\tau}(w)=\left\{k \in K: k w \notin V_{\tau}^{<}\right\} .
$$

Lemma 3. For every $w \in G \cdot w^{+}$,

$$
\lim _{\tau \rightarrow 0^{+}} \nu\left(K-K_{\tau}(w)\right)=0
$$

Proof. It follows from the Iwasawa decomposition that $G \cdot w^{+}=K \cdot w^{+}$. Thus, without loss of generality, we may assume that $w=w^{+}$. By the continuity of the measure, it suffices to prove that

$$
\nu\left(\left\{k \in K: k w^{+} \in V^{<}\right\}\right)=0 .
$$

Suppose that this is false. For a subspace $W$ of $V$, define

$$
K_{W}=\left\{k \in K: k w^{+} \in W\right\} .
$$


Let $W$ be a minimal subspace of $V^{<}$such that $\nu\left(K_{W}\right)>0$. We claim that $\operatorname{Stab}_{K}(W)=$ $K$. If $\operatorname{Stab}_{K}(W)$ has infinite index in $K$, then there exist $k_{i} \in K, i \geq 1$, such that $k_{i} W \neq k_{j} W$ for $i \neq j$. Since all sets $k_{i} K_{W} \subset K, i \geq 1$, have the same positive measure, it follows that for some $i \neq j, k_{i} K_{W} \cap k_{j} K_{W}$ has positive measure. Then $k_{j}^{-1} k_{i} K_{W} \cap K_{W}$ has positive measure too, and for $k \in k_{j}^{-1} k_{i} K_{W} \cap K_{W}$,

$$
k w^{+} \in k_{j}^{-1} k_{i} W \cap W .
$$

Since $k_{j}^{-1} k_{i} W \cap W$ is a proper subspace of $W$, this contradicts the choice of $W$. Thus, $\operatorname{Stab}_{K}(W)$ is a closed subgroup of finite index in $K$. Since $K$ is connected, it follows that $K=\operatorname{Stab}_{K}(W)$. Then $w^{+} \in K_{W}^{-1} W \subset V^{<}$. This contradiction proves the lemma.

We consider the sets

$$
\begin{aligned}
A_{T}^{\eta} & =\left\{a \in A_{T}: \alpha(\log a) \geq \eta \text { for all } \alpha \in \Sigma^{+}\right\} \\
G_{T, \varepsilon}(u, v) & =\left\{g \in G_{T}(\tilde{x}): \tilde{d}(g u, g v)>\varepsilon\right\} \\
\Omega_{T, \tau}^{\eta}(u, v) & =\tilde{g}^{-1} K A_{T}^{\eta}\left(K_{\tau}(u) \cap K_{\tau}(v)\right)
\end{aligned}
$$

defined for $T, \eta, \tau, \varepsilon>0$ and $u, v \in \mathbb{P}(V)$.

Lemma 4. For every $\varepsilon>0$ and $\tau>0$, there exists $\eta>0$ such that for every $T>0$ and $u, v \in G \cdot w^{+}$,

$$
\Omega_{T, \tau}^{\eta}(u, v) \cap G_{T, \varepsilon}(u, v)=\emptyset .
$$

Proof. Note that an element $a \in A_{T}^{\eta}$ acts by diagonal transformations on $V$ with respect to some fixed basis, and the eigenvalue associated to the vector $w^{+}$is at least $e^{\eta}$ times greater than the other eigenvalues. Therefore, for all $w \notin V_{\tau}^{<}$and sufficiently large $\eta$ (depending only on $\tau$ and $\varepsilon$ ), we have $d\left(a w, w^{+}\right)<\varepsilon / 2$ when $a \in A_{T}^{\eta}$. Thus, for

$$
\tilde{g}^{-1} k_{1} a k_{2} \in \Omega_{T, \tau}^{\eta}(u, v)=\tilde{g}^{-1} K A_{T}^{\eta}\left(K_{\tau}(u) \cap K_{\tau}(v)\right),
$$

we have

$$
\tilde{d}\left(\tilde{g}^{-1} k_{1} a k_{2} u, \tilde{g}^{-1} k_{1} a k_{2} v\right)=d\left(a k_{2} u, a k_{2} v\right) \leq d\left(a k_{2} u, w^{+}\right)+d\left(a k_{2} v, w^{+}\right)<\varepsilon,
$$

This proves the lemma.

2.4. Completion of the proof. By (3),

$$
\operatorname{Vol}\left(\Omega_{T, \tau}^{\eta}(u, v)\right)=\left(\int_{A_{T}^{\eta}} \xi(\log a) d a\right) \cdot \nu\left(K_{\tau}(u) \cap K_{\tau}(v)\right) .
$$

Let $\varepsilon, \delta \in(0,1)$. Using Lemma 3, we choose $\tau>0$ such that

$$
\nu\left(K_{\tau}(u) \cap K_{\tau}(v)\right)>1-\delta .
$$


Let $\eta>0$ be as Lemma 4. By Lemma 9(a), for sufficiently large $T$,

$$
\int_{A_{T}^{\eta}} \xi(a) d a \geq(1-\delta) \int_{A_{T}^{+}} \xi(\log a) d a .
$$

Thus, it follows from (41) and (7) that

$$
\operatorname{Vol}\left(\Omega_{T, \tau}^{\eta}(u, v)\right) \geq(1-\delta)^{2} \operatorname{Vol}\left(G_{T}(\tilde{x})\right) .
$$

for sufficiently large $T>0$. Therefore, by (6),

$$
\operatorname{Vol}\left(G_{T, \varepsilon}(u, v)\right) \leq\left(1-(1-\delta)^{2}\right) \operatorname{Vol}\left(G_{T}(\tilde{x})\right)
$$

for all $\delta \in(0,1)$ and sufficiently large $T>0$. Since the sets

$$
\left\{\left(g_{1} P, g_{2} P\right): \tilde{d}\left(g_{1} w^{+}, g_{2} w^{+}\right)<\varepsilon\right\}, \quad \varepsilon>0,
$$

form a base of the neighborhoods of the diagonal in $G / P \times G / P$, this proves the first part of Theorem 2 .

To prove the second part of Theorem 2, we choose a neighborhood $\mathcal{P}$ of $e$ in $G$ and a neighborhood $\mathcal{Q}$ of the diagonal in $G / P \times G / P$ such that

$$
\begin{aligned}
\mathcal{P}^{-1} \mathcal{P} \cap \Gamma & =\{e\}, \\
\mathcal{P}^{-1} \cdot \mathcal{Q} & \subset \mathcal{O}, \\
\mathcal{P} \cdot G_{T}(\tilde{x}) & \subset G_{T+c}(\tilde{x}) .
\end{aligned}
$$

for fixed $c>0$ and all $T>0$. Here we use that $\Gamma$ is discrete, the space $G / P$ is compact, and the metric on the symmetric space is uniformly continuous. By (9), for every $\gamma \in \Gamma$ such that $\gamma \cdot(u, v) \notin \mathcal{O}$, we have $\mathcal{P} \gamma \cdot(u, v) \cap \mathcal{Q}=\emptyset$. Thus, using (10), we deduce that

$$
\mathcal{P} \cdot\left\{\gamma \in \Gamma_{T}(\tilde{x}): \gamma \cdot(u, v) \notin \mathcal{O}\right\} \subset\left\{g \in G_{T+c}(\tilde{x}): g \cdot(u, v) \notin \mathcal{Q}\right\} .
$$

Then by (8), $\mathcal{P} \gamma_{1} \cap \mathcal{P} \gamma_{2}=\emptyset$ for $\gamma_{1}, \gamma_{2} \in \Gamma, \gamma_{1} \neq \gamma_{2}$, and

$$
\begin{aligned}
\left|\left\{\gamma \in \Gamma_{T}(\tilde{x}): \gamma \cdot(u, v) \notin \mathcal{O}\right\}\right| & \leq \frac{1}{\operatorname{Vol}(\mathcal{P})} \operatorname{Vol}\left(\left\{g \in G_{T+c}(\tilde{x}): g \cdot(u, v) \notin \mathcal{Q}\right\}\right) \\
& =o\left(\operatorname{Vol}\left(G_{T+c}(\tilde{x})\right)\right)
\end{aligned}
$$

as $T \rightarrow \infty$. Now the second statement of Theorem 2 follows from Lemma 9(d) and (11).

\section{Equidistribution ON $\Gamma \backslash G$}

Recall that $K$ is a maximal compact subgroups of $G$, and $\nu$ is the probability Haar measure on $K$. Denote by $\varrho$ a right Haar measure on the minimal parabolic subgroup $P$. For a suitable normalization of $\varrho$, the Haar measure on $G$ is given by

$$
\int_{G} \psi(g) d g=\int_{K} \int_{P} \psi(k p) d \varrho(p) d \nu(k), \quad \psi \in C_{c}(G) .
$$


We also define a measure $\mu$ on $G$ by

$$
\int_{G} \psi(g) d \mu(g)=\int_{K} \int_{P} \psi\left(k p^{-1}\right) d \varrho(p) d \nu(k), \quad \psi \in C_{c}(G) .
$$

Note that $\mu$ is left $K$-invariant.

The first step in the proof of Theorem 1 is the following result.

Proposition 5. For every $\Psi \in C_{c}(\Gamma \backslash G)$ and $z \in \Gamma \backslash G$,

$$
\lim _{T \rightarrow \infty} \frac{1}{\mu\left(G_{T}\right)} \int_{G_{T}} \Psi(z g) d \mu(g)=\frac{1}{\operatorname{Vol}(\Gamma \backslash G)} \int_{\Gamma \backslash G} \Psi d g
$$

where $G_{T}=\{g \in G: d(x, x g)<T\}$.

Proposition 5 is a consequence of the equidistribution of translates of $K$ in $\Gamma \backslash G$ proved by Eskin and McMullen in EM] (see also [S] for a more general result). They showed that for every strongly divergent sequence $\left\{g_{n}\right\} \subset G$,

$$
\lim _{n \rightarrow \infty} \int_{K} \Psi\left(z k g_{n}\right) d \nu(k)=\frac{1}{\operatorname{Vol}(\Gamma \backslash G)} \int_{\Gamma \backslash G} \Psi d g .
$$

Recall that a sequence $\left\{g_{n}\right\} \subset G$ is strongly divergent if the projection of $\left\{g_{n}\right\}$ on every noncompact simple factor of $G$ is divergent. Note that (13) was proved in EM] under the condition that the lattice $\Gamma$ is irreducible. Since the proof of (13) is based on mixing properties of the action of $G$ on $\Gamma \backslash G$, it is applicable to the case of a reducible lattice $\Gamma$ provided that the sequence $\left\{g_{n}\right\}$ is strongly divergent.

Denote by $\pi_{i}: G \rightarrow G_{i}, i=1, \ldots, s$, the projections of $G$ onto its simple factors. Let $C_{i, j} \subset G_{i}, j \geq 1$, be an increasing sequence of compact subsets such that $G_{i}=$ $\cup_{j \geq 1} C_{i, j}$. Define

$$
G_{T, n}=G_{T}-\bigcup_{1 \leq i \leq s} \pi_{i}^{-1}\left(C_{i, n}\right) .
$$

Lemma 6. For every $n \geq 1, \mu\left(G_{T, n}\right) \sim \mu\left(G_{T}\right)$ as $T \rightarrow \infty$.

Proof. It suffices to show that for every $i=1, \ldots, s$ and $n \geq 1$,

$$
\mu\left(G_{T} \cap \pi_{i}^{-1}\left(C_{i, n}\right)\right)=o\left(\mu\left(G_{T}\right)\right) \text { as } T \rightarrow \infty .
$$

Fix $i=1, \ldots, s$ and $n \geq 1$. Note that $G=D H$, where $D$ and $H=\operatorname{ker}\left(\pi_{i}\right)$ are normal connected semisimple Lie subgroups with finite centers, and $D$ and $H$ commute. We have $\pi_{i}^{-1}\left(C_{i, n}\right)=D_{i, n} H$ for some compact set $D_{i, n} \subset D$. There is a constant $\delta>0$ such that

$$
D_{i, n} H_{T-\delta} \subset\left(D_{i, n} H\right)_{T} \subset D_{i, n} H_{T+\delta} \text { for all } T>0 .
$$

We define measures $\mu_{D}$ and $\mu_{H}$ for the groups $D$ and $H$ respectively as in (12). With appropriate normalization, $\mu=\mu_{D} \otimes \mu_{H}$. Thus, it follows from (15) that

$$
\mu\left(G_{T} \cap \pi_{i}^{-1}\left(C_{i, n}\right)\right)=\mu\left(\left(D_{i, n} H\right)_{T}\right) \ll \mu_{H}\left(H_{T+\delta}\right) .
$$


Since $G_{T}=K P_{T}$ and $P_{T}^{-1}=P_{T}$, using (11) and (12), we conclude that

$$
\mu\left(G_{T}\right)=\varrho\left(P_{T}^{-1}\right)=\varrho\left(P_{T}\right)=\operatorname{Vol}\left(G_{T}\right)
$$

Similarly, $H=L Q_{T}$ where $L$ is a maximal compact subgroup of $H$ contained in $K$, and $Q$ is a minimal parabolic subgroup of $H$. As in (17), we deduce that $\mu_{H}\left(H_{T}\right)=$ $\operatorname{Vol}_{H}\left(H_{T}\right)$. By (16), it is sufficient to show that

$$
\operatorname{Vol}_{H}\left(H_{T+\delta}\right)=o\left(\operatorname{Vol}\left(G_{T}\right)\right) \quad \text { as } \quad T \rightarrow \infty
$$

Note that with appropriate normalization the Haar measure on $G$ is the product of Haar measures on $D$ and $H$. Without loss of generality, $\operatorname{Vol}_{D}\left(D_{i, n}\right)>0$. Then by (15),

$$
\operatorname{Vol}_{H}\left(H_{T+\delta}\right) \ll \operatorname{Vol}\left(D_{i, n} H_{T+\delta}\right) \leq \operatorname{Vol}\left(\left(D_{i, n} H\right)_{T+2 \delta}\right)
$$

Let $G_{T}^{\eta}$ be defined as in (24). Since the set $D_{i, n}$ is compact, there exists $\eta>0$ such that

$$
\left(D_{i, n} H\right)_{T+2 \delta} \subset G_{T+2 \delta}-G_{T+2 \delta}^{\eta}
$$

Thus, (18) follows from Lemma 9(b).

Proof of Proposition 5. The map $K \times A^{+} \times K \rightarrow G$ is a diffeomorphism on an open set of full measure. Since the measure $\mu$ is left $K$-invariant and smooth, for some $\sigma \in C\left(A^{+} \times K\right)$

$$
\int_{G} \psi(g) d \mu(g)=\int_{K} \int_{A^{+}} \int_{K} \psi\left(k_{1} a k_{2}\right) \sigma\left(a, k_{2}\right) d \nu\left(k_{1}\right) d a d \nu\left(k_{2}\right), \quad \psi \in C_{c}(G) .
$$

Let $G_{T, n}$ be defined as in (14), and it is $K$-bi-invariant (equivalently, all $C_{i, j}$ are $\pi_{i}(K)$-bi-invariant). Then

$$
G_{T, n}=K A_{T, n}^{+} K \text { and } \mu\left(G_{T, n}\right)=\int_{K} \int_{A_{T, n}^{+}} \sigma\left(a, k_{2}\right) d a d \nu\left(k_{2}\right)
$$

where $A_{T, n}^{+}=G_{T, n} \cap A^{+}$.

Let $\varepsilon>0$. By (13),

$$
\left|\int_{K} \Psi\left(z k_{1} a k_{2}\right) d \nu\left(k_{1}\right)-\frac{1}{\operatorname{Vol}(\Gamma \backslash G)} \int_{\Gamma \backslash G} \Psi d g\right|<\varepsilon
$$


for $a \in A_{T, n}^{+}$and $k_{2} \in K$ when $n>n_{0}(\varepsilon)$. Thus, for $n>n_{0}(\varepsilon)$,

$$
\begin{aligned}
& \left|\int_{G_{T, n}} \Psi(z g) d \mu(g)-\frac{\mu\left(G_{T, n}\right)}{\operatorname{Vol}(\Gamma \backslash G)} \int_{\Gamma \backslash G} \Psi d g\right| \\
= & \mid \int_{K} \int_{A_{T, n}^{+}} \int_{K} \Psi\left(z k_{1} a k_{2}\right) d \nu\left(k_{1}\right) \sigma\left(a, k_{2}\right) d a d \nu\left(k_{2}\right) \\
- & \frac{\mu\left(G_{T, n}\right)}{\operatorname{Vol}(\Gamma \backslash G)} \int_{\Gamma \backslash G} \Psi d g\left|\leq \int_{K} \int_{A_{T, n}^{+}}\right| \int_{K} \Psi\left(z k_{1} a k_{2}\right) d \nu\left(k_{1}\right) \\
- & \frac{1}{\operatorname{Vol}(\Gamma \backslash G)} \int_{\Gamma \backslash G} \Psi d g \mid \sigma\left(a, k_{2}\right) \operatorname{dad\nu }\left(k_{2}\right)<\varepsilon \mu\left(G_{T, n}\right) .
\end{aligned}
$$

By Lemma 6, for every $n \geq 1$,

$$
\int_{G_{T}} \Psi(z g) d \mu(g)=\int_{G_{T, n}} \Psi(z g) d \mu(g)+o\left(\mu\left(G_{T, n}\right)\right)
$$

as $T \rightarrow \infty$. Thus, it follows from (19) that

$$
\limsup _{T \rightarrow \infty}\left|\frac{1}{\mu\left(G_{T}\right)} \int_{G_{T}} \Psi(z g) d \mu(g)-\frac{1}{\operatorname{Vol}(\Gamma \backslash G)} \int_{\Gamma \backslash G} \Psi d g\right|<\varepsilon
$$

for every $\varepsilon>0$. This proves the proposition.

\section{Equidistribution on AVERAGE}

In this section we prove that Theorem 1 holds "on average". In the case of hyperbolic spaces, the following proposition is a consequence of the work of Roblin $[\mathrm{R}]$.

Proposition 7. For every $f \in C(G / P)$ and $y \in G / P$,

$$
\lim _{T \rightarrow \infty} \frac{1}{\left|\Gamma_{T}(\tilde{x})\right|} \sum_{\gamma \in \Gamma_{T}(\tilde{x})} \int_{K} f(\gamma k y) d \nu(k)=\int_{G / P} f d m_{\tilde{x}}
$$

where $\Gamma_{T}(\tilde{x})=\{\gamma \in \Gamma: d(x, \tilde{x} \gamma)<T\}$.

Proof. There exists $\tilde{p} \in P$ such that $\tilde{x}=x \tilde{p}$. Then $\tilde{K}=\tilde{p}^{-1} K \tilde{p}$, and it follows from (11) that

$$
\int_{G} \psi(g) d g=\int_{\tilde{K}} \int_{P} \psi\left(k \tilde{p}^{-1} p\right) d \varrho(p) d \tilde{\nu}(k), \quad \psi \in C_{c}(G) .
$$

Without loss of generality, $f \geq 0$, and since $G=K P$, we may assume that $y=e P$. Let $\varepsilon>0, \mathcal{O}_{\varepsilon}=\{z \in X: d(x, z)<\varepsilon\}$, and $\phi_{\varepsilon} \in C_{c}(X)$ such that

$$
\phi_{\varepsilon} \geq 0, \quad \operatorname{supp}\left(\phi_{\varepsilon}\right) \subset \mathcal{O}_{\varepsilon}, \quad \int_{P} \phi_{\varepsilon}\left(x p^{-1}\right) d \varrho(p)=1 .
$$


Since $X=\tilde{x} P$ and $\varrho$ is right invariant, it follows that

$$
\int_{P} \phi_{\varepsilon}\left(z p^{-1}\right) d \varrho(p)=1 \quad \text { for every } z \in X .
$$

Let

$$
\psi_{\varepsilon}(g)=f(g P) \phi_{\varepsilon}(\tilde{x} g), \quad g \in G .
$$

Clearly, $\psi_{\varepsilon} \in C_{c}(G)$ and

$$
\Psi_{\varepsilon}(\Gamma g) \stackrel{\text { def }}{=} \sum_{\gamma \in \Gamma} \psi_{\varepsilon}(\gamma g) \in C_{c}(\Gamma \backslash G) .
$$

By Proposition 5 ,

$$
\lim _{T \rightarrow \infty} \frac{1}{\mu\left(G_{T}\right)} \sum_{\gamma \in \Gamma} \int_{G_{T}} \psi_{\varepsilon}(\gamma g) d \mu(g)=\frac{1}{\operatorname{Vol}(\Gamma \backslash G)} \int_{\Gamma \backslash G} \Psi_{\varepsilon}(\Gamma g) d g
$$

and by (20),

$$
\begin{aligned}
\operatorname{Vol}(\Gamma \backslash G) \int_{\Gamma \backslash G} \Psi_{\varepsilon}(\Gamma g) d g & =\int_{G} \psi_{\varepsilon}(g) d g=\int_{\tilde{K}} f(k P) d \tilde{\nu}(k) \cdot \int_{P} \phi_{\varepsilon}\left(\tilde{x} \tilde{p}^{-1} p\right) d \varrho(p) \\
& =\int_{G / P} f d m_{\tilde{x}} \cdot \int_{P} \phi_{\varepsilon}(x p) d \varrho(p) .
\end{aligned}
$$

Denote by $\delta$ the modular function of $P$. By (21),

$$
\begin{aligned}
\left|\int_{P} \phi_{\varepsilon}(x p) d \varrho(p)-1\right| & =\left|\int_{P} \phi_{\varepsilon}\left(x p^{-1}\right)(\delta(p)-1) d \varrho(p)\right| \\
& \leq \max \left\{|\delta(p)-1|: x p^{-1} \in \mathcal{O}_{\varepsilon}\right\} .
\end{aligned}
$$

The sets $\left\{p \in P: x p^{-1} \in \mathcal{O}_{\varepsilon}\right\}, \varepsilon>0$, form a base of neighborhoods of $P \cap K$ in $P$. Since $\left.\delta\right|_{P \cap K}=1$ and $P \cap K$ is compact,

$$
\max \left\{|\delta(p)-1|: x p^{-1} \in \mathcal{O}_{\varepsilon}\right\} \rightarrow 0 \quad \text { as } \varepsilon \rightarrow 0^{+} .
$$

Thus, it follows from (22) that

$$
\lim _{\varepsilon \rightarrow 0^{+}} \lim _{T \rightarrow \infty} \frac{1}{\mu\left(G_{T}\right)} \sum_{\gamma \in \Gamma} \int_{G_{T}} \psi_{\varepsilon}(\gamma g) d \mu(g)=\int_{G / P} f d m_{\tilde{x}} .
$$

Since $G_{T}=K P_{T}$,

$$
\begin{aligned}
& \sum_{\gamma \in \Gamma} \int_{G_{T}} \psi_{\varepsilon}(\gamma g) d \mu(g) \\
\stackrel{\text { 12. }}{=} & \sum_{\gamma \in \Gamma} \int_{K \times P_{T}^{-1}} \psi_{\varepsilon}\left(\gamma k p^{-1}\right) d \nu(k) d \varrho(p) \\
= & \sum_{\gamma \in \Gamma} \int_{K} f(\gamma k P)\left(\int_{P_{T}^{-1}} \phi_{\varepsilon}\left(\tilde{x} \gamma k p^{-1}\right) d \varrho(p)\right) d \nu(k) .
\end{aligned}
$$


For $\gamma \in \Gamma-\Gamma_{T+\varepsilon}(\tilde{x}), k \in K$, and $p \in P_{T}^{-1}$,

$$
d\left(x, \tilde{x} \gamma k p^{-1}\right)=d\left(x p k^{-1}, \tilde{x} \gamma\right) \geq d(x, \tilde{x} \gamma)-d\left(x, x p k^{-1}\right) \geq \varepsilon .
$$

This implies that $\int_{P_{T}^{-1}} \phi_{\varepsilon}\left(\tilde{x} \gamma k p^{-1}\right) d \varrho(p)=0$ for $\gamma \in \Gamma-\Gamma_{T+\varepsilon}(\tilde{x})$. Thus,

$$
\begin{aligned}
& \sum_{\gamma \in \Gamma} \int_{G_{T}} \psi_{\varepsilon}(\gamma g) d \mu(g) \\
= & \sum_{\gamma \in \Gamma_{T+\varepsilon}(\tilde{x})} \int_{K} f(\gamma k P)\left(\int_{P_{T}^{-1}} \phi_{\varepsilon}\left(\tilde{x} \gamma k p^{-1}\right) d \varrho(p)\right) d \nu(k) \\
\leq & \sum_{\gamma \in \Gamma_{T+\varepsilon}(\tilde{x})} \int_{K} f(\gamma k P)\left(\int_{P} \phi_{\varepsilon}\left(\tilde{x} \gamma k p^{-1}\right) d \varrho(p)\right) d \nu(k) \\
\underline{\text { 21] }} & \sum_{\gamma \in \Gamma_{T+\varepsilon}(\tilde{x})} \int_{K} f(\gamma k P) d \nu(k) .
\end{aligned}
$$

Combining (23), (17), (11) and Lemma 9)(c), we deduce that

$$
\liminf _{T \rightarrow \infty} \frac{1}{\left|\Gamma_{T}(\tilde{x})\right|} \sum_{\gamma \in \Gamma_{T}(\tilde{x})} \int_{K} f(\gamma k P) d \nu(k) \geq \int_{G / P} f d m_{\tilde{x}} .
$$

On the other hand, for $\gamma \in \Gamma_{T-\varepsilon}(\tilde{x}), k \in K$, and $p \in P$ such that $d\left(x, \tilde{x} \gamma k p^{-1}\right)<\varepsilon$,

$$
d\left(x, x p^{-1}\right) \leq d\left(x, \tilde{x} \gamma k p^{-1}\right)+d\left(x p^{-1}, \tilde{x} \gamma k p^{-1}\right)<T .
$$

This shows that for $\gamma \in \Gamma_{T-\varepsilon}(\tilde{x})$,

$$
\int_{P_{T}^{-1}} \phi_{\varepsilon}\left(\tilde{x} \gamma k p^{-1}\right) d \varrho(p)=\int_{P} \phi_{\varepsilon}\left(\tilde{x} \gamma k p^{-1}\right) d \varrho(p) \stackrel{21]}{=} 1 .
$$

Hence,

$$
\begin{aligned}
& \sum_{\gamma \in \Gamma} \int_{G_{T}} \psi_{\varepsilon}(\gamma g) d \mu(g) \\
\geq & \sum_{\gamma \in \Gamma_{T-\varepsilon}(\tilde{x})} \int_{K} f(\gamma k P)\left(\int_{P_{T}^{-1}} \phi_{\varepsilon}\left(\tilde{x} \gamma k p^{-1}\right) d \varrho(p)\right) d \nu(k) \\
= & \sum_{\gamma \in \Gamma_{T-\varepsilon}(\tilde{x})} \int_{K} f(\gamma k P) d \nu(k) .
\end{aligned}
$$

By (23), (17), (11), and Lemma 9(c),

$$
\limsup _{T \rightarrow \infty} \frac{1}{\left|\Gamma_{T}(\tilde{x})\right|} \sum_{\gamma \in \Gamma_{T}(\tilde{x})} \int_{K} f(\gamma k P) d \nu(k) \leq \int_{G / P} f d m_{\tilde{x}}
$$

This proves the proposition. 


\section{Proof of Theorem [1]}

Now the proof can be completed using the argument from $\mathrm{Ma}$. Let $\varepsilon>0$. Since the space $G / P \times G / P$ is compact, there exists a neighborhood $\mathcal{O}$ of the diagonal in $G / P \times G / P$ such that for every $\left(z_{1}, z_{2}\right) \in \mathcal{O}$, we have $\left|f\left(z_{1}\right)-f\left(z_{2}\right)\right|<\varepsilon$. Then for every $k \in K$,

$$
\begin{aligned}
& \left|\sum_{\gamma \in \Gamma_{T}(\tilde{x})} f(\gamma y)-\sum_{\gamma \in \Gamma_{T}(\tilde{x})} f(\gamma k y)\right| \\
& \leq \sum_{\gamma \in \Gamma_{T}(\tilde{x}):(\gamma y, \gamma k y) \in \mathcal{O}}|f(\gamma y)-f(\gamma k y)|+\sum_{\gamma \in \Gamma_{T}(\tilde{x}):(\gamma y, \gamma k y) \notin \mathcal{O}}|f(\gamma y)-f(\gamma k y)| \\
& \leq \varepsilon\left|\Gamma_{T}(\tilde{x})\right|+2 \sup |f| \cdot\left|\left\{\gamma \in \Gamma_{T}(\tilde{x}):(\gamma y, \gamma k y) \notin \mathcal{O}\right\}\right| .
\end{aligned}
$$

Thus, it follows from Theorem 2 that

$$
\lim _{T \rightarrow \infty} \frac{1}{\left|\Gamma_{T}(\tilde{x})\right|}\left|\sum_{\gamma \in \Gamma_{T}(\tilde{x})} f(\gamma y)-\sum_{\gamma \in \Gamma_{T}(\tilde{x})} f(\gamma k y)\right|=0
$$

for all $k \in K$. Hence, by the dominated convergence theorem,

$$
\lim _{T \rightarrow \infty}\left|\frac{1}{\left|\Gamma_{T}(\tilde{x})\right|} \sum_{\gamma \in \Gamma_{T}(\tilde{x})} f(\gamma y)-\frac{1}{\left|\Gamma_{T}(\tilde{x})\right|} \sum_{\gamma \in \Gamma_{T}(\tilde{x})} \int_{K} f(\gamma k y) d \nu(k)\right|=0 .
$$

Finally, Theorem 1 follows from Proposition 7.

\section{Appendix: volume estimates}

In this section, we give proofs of volume estimates, which are used in Theorems 1 and 2 There are other ways to establish these volume estimates. For example, one can use the exact asymptotics of the volume of Riemannian balls from [Kn] (see also GO]). We present a straightforward proof that does not use asymptotics.

Let $\mathfrak{a}$ be the Lie algebra of the Cartan subgroup $A$ and $\mathfrak{a}^{+}$the positive Weyl chamber with respect to the root system $\Sigma^{+}$. The Riemannian metric defines a scalar product on $\mathfrak{a}$ and, by duality, on the dual space of $\mathfrak{a}$. For $\alpha \in \Sigma^{+}$, we denote by $m_{\alpha}$ the dimension of the corresponding root space and put $\rho=\frac{1}{2} \sum_{\beta \in \Sigma^{+}} m_{\beta} \beta$.

Lemma 8. The maximum of $\rho$ on $\{a \in \mathfrak{a}:\|a\| \leq 1\}$ is achieved at a unique point in the interior of $\mathfrak{a}^{+}$.

Proof. Since the set $\{a \in \mathfrak{a}:\|a\|=1\}$ is strictly convex, it is clear that the point of maximum is unique. It is sufficient to show that $(\rho, \alpha)>0$ for every $\alpha \in \Sigma^{+}$. Denote by $\sigma_{\alpha}$ the reflection with respect to the hyperplane $\{\alpha=0\}$. The map $\sigma_{\alpha}$ permutes the elements of the set $\Sigma^{+}-\{\alpha, 2 \alpha\}$ and $\sigma_{\alpha}(\alpha)=-\alpha$. Since $m_{\sigma_{\alpha}(\beta)}=m_{\beta}$, we have

$$
\sigma_{\alpha}(\rho)=\rho-2 m_{\alpha} \alpha-4 m_{2 \alpha} \alpha \text {. }
$$


Thus,

$$
(\rho, \alpha)=\left(\sigma_{\alpha}(\rho), \sigma_{\alpha}(\alpha)\right)=2 m_{\alpha}(\alpha, \alpha)+4 m_{2 \alpha}(\alpha, \alpha)-(\rho, \alpha)
$$

and $(\rho, \alpha)=\left(m_{\alpha}+2 m_{2 \alpha}\right)(\alpha, \alpha)$ is positive.

For $T, \eta>0$, define

$$
\begin{aligned}
A_{T}^{\eta} & =\left\{a \in A_{T}: \alpha(\log a) \geq \eta \text { for all } \alpha \in \Sigma^{+}\right\} \\
& =\left\{a \in A:\|\log a\|<T, \alpha(\log a) \geq \eta \text { for all } \alpha \in \Sigma^{+}\right\}, \\
G_{T}^{\eta} & =K A_{T}^{\eta} K .
\end{aligned}
$$

Lemma 9. For every $\eta>0$,

(a)

(c)

$$
\int_{A_{T}^{\eta}} \xi(\log a) d a \sim_{T \rightarrow \infty} \int_{A_{T}^{+}} \xi(\log a) d a
$$

$$
\operatorname{Vol}\left(G_{T}^{\eta}\right) \sim_{T \rightarrow \infty} \operatorname{Vol}\left(G_{T}\right)
$$

$$
\liminf _{\varepsilon \rightarrow 0^{+}}\left(\limsup _{T \rightarrow \infty} \frac{\operatorname{Vol}\left(G_{T+\varepsilon}\right)}{\operatorname{Vol}\left(G_{T}\right)}\right)=1,
$$

$$
\operatorname{Vol}\left(G_{T+\eta}\right) \ll \operatorname{Vol}\left(G_{T}\right)
$$

Proof. We have

$$
\int_{\mathfrak{a}_{T}^{+}} \xi(a) d a=2^{-\left|\Sigma^{+}\right|} \sum_{i \in I} \int_{\mathfrak{a}_{T}^{+}} e^{\lambda_{i}(a)} d a
$$

where $\lambda_{i}$ 's the characters of the form $2 \rho-\sum_{\alpha \in \Sigma^{+}} n_{\alpha} \alpha$ for some $n_{\alpha} \geq 0$. Let

$$
\begin{aligned}
\delta & =\max \left\{2 \rho(a): a \in \mathfrak{a}_{1}^{+}\right\}, \\
\delta_{i} & =\max \left\{\lambda_{i}(a): a \in \mathfrak{a}_{1}^{+}\right\}, \quad i \in I, \\
\delta_{\alpha} & =\max \left\{2 \rho(a): a \in \mathfrak{a}_{1}^{+}, \alpha(a)=0\right\}, \quad \alpha \in \Sigma^{+} .
\end{aligned}
$$

It follows from Lemma 8 that for $\lambda_{i} \neq 2 \rho$ and $\alpha \in \Sigma^{+}, \delta>\max \left\{\delta_{i}, \delta_{\alpha}\right\}$. Thus,

$$
\int_{\mathfrak{a}_{T}^{+}} e^{\lambda_{i}(a)} d a \leq \operatorname{Vol}\left(\mathfrak{a}_{T}^{+}\right) e^{\delta_{i} T} \ll T^{r} e^{\delta_{i} T}
$$

where $r=\operatorname{dim} \mathfrak{a}$. Let $\varepsilon>0$ be such that

$$
\delta-\varepsilon>\max \left\{\delta_{i}, \delta_{\alpha}: i \in I, \alpha \in \Sigma^{+}\right\} \text {. }
$$

Then

$$
\begin{aligned}
\int_{\mathfrak{a}_{T}^{+}} e^{2 \rho(a)} d a & =T^{r} \int_{\mathfrak{a}_{1}^{+}} e^{2 T \rho(a)} d a \\
& \geq T^{r} e^{(\delta-\varepsilon) T} \operatorname{Vol}\left(\left\{a \in \mathfrak{a}_{1}^{+}: 2 \rho(a) \geq \delta-\varepsilon\right\}\right) \gg T^{r} e^{(\delta-\varepsilon) T} .
\end{aligned}
$$


Combining (25), (26), and (27) , we deduce that

$$
\int_{\mathfrak{a}_{T}^{+}} \xi(a) d a \gg T^{r} e^{(\delta-\varepsilon) T} .
$$

On the other hand, for $\alpha \in \Sigma^{+}$,

$$
\begin{aligned}
\int_{\mathfrak{a}_{T}^{+} \cap\{\alpha<\eta\}} \xi(a) d a & \leq \int_{\mathfrak{a}_{T}^{+} \cap\{\alpha<\eta\}} e^{2 \rho(a)} d a \ll \int_{\mathfrak{a}_{T}^{+} \cap\{\alpha=0\}} e^{2 \rho(a)} d a \\
& =T^{r-1} \int_{\mathfrak{a}_{1}^{+} \cap\{\alpha=0\}} e^{2 T \rho(a)} d a \ll T^{r-1} e^{\delta_{\alpha} T}=o\left(e^{(\delta-\varepsilon) T}\right) .
\end{aligned}
$$

Since

$$
\mathfrak{a}_{T}^{+}-\mathfrak{a}_{T}^{\eta} \subset \bigcup_{\alpha \in \Sigma^{+}} \mathfrak{a}_{T}^{+} \cap\{\alpha<\eta\} .
$$

This proves part (a) of the lemma. Part (b) follows from (2).

To prove part (c), we note that

$$
\operatorname{Vol}\left(G_{T+\varepsilon}\right)=\int_{\mathfrak{a}_{T+\varepsilon}^{+}} \xi(a) d a=(T+\varepsilon)^{r} \int_{\mathfrak{a}_{1}^{+}} \xi((T+\varepsilon) a) d a
$$

It is easy to check that there exist $b>0$ such that $\sinh (t+\varepsilon) \leq e^{\varepsilon} \sinh (t)+b$ for every $\varepsilon \in(0,1)$ and $t \geq 0$. Thus, for $a \in \mathfrak{a}_{1}^{+}$and sufficiently small $\varepsilon>0$,

$$
\xi((T+\varepsilon) a) \leq \prod_{\alpha \in \Sigma^{+}}\left(a_{\varepsilon} \sinh (\alpha(T a))+b\right)^{m_{\alpha}} \leq d_{\varepsilon} \xi(T a)+C \sum_{i \in I} e^{\lambda_{i}(a)}
$$

where $d_{\varepsilon} \rightarrow 1$ as $\varepsilon \rightarrow 0^{+}, C>0$, and $\lambda_{i}$ 's are characters such that $2 \rho-\lambda_{i}<0$ in the interior of $\mathfrak{a}^{+}$. Thus, it follows from (26) that

$$
\int_{\mathfrak{a}_{T}^{+}} \xi((T+\varepsilon) a) d a \leq d_{\varepsilon} \int_{\mathfrak{a}_{T}^{+}} \xi(T a) d a+o\left(e^{(\delta-\varepsilon) T}\right) .
$$

Using (41) and (28), we deduce that

$$
\limsup _{T \rightarrow \infty} \frac{\operatorname{Vol}\left(G_{T+\varepsilon}\right)}{\operatorname{Vol}\left(G_{T}\right)} \leq d_{\varepsilon}
$$

and part (c) of the lemma follows. The last part of lemma can be proved similarly.

\section{AcKnowledgements}

The main ideas of this paper were developed during the workshop "Ergodic properties of geometric group actions" in Summer 2003. The authors would like to express deep appreciation to the organizers of this workshop and to the Max Planck Institute of Mathematics for its support. We also would like to thank to R. Spatzier for raising the problem solved in this paper during the workshop and to Y. Guivarc'h for bringing the paper of Arnold and Krylov to out attention. 


\section{REFERENCES}

[AK] V. I. Arnold and A. L. Krylov, Uniform distribution of points on a sphere and certain ergodic properties of solutions of linear ordinary differential equations in a complex domain. Dokl. Akad. Nauk SSSR 148, 9-12, 1963.

[DRS] W. Duke,Z. Rudnick and P. Sarnak, Density of integer points on affine homogeneous varieties. Duke Math. J. 71 (1993), no. 1, 143-179.

[EM] A. Eskin and C. McMullen, Mixing, counting and equidistribution on Lie groups. Duke Math. J. 71 (1993), no. 1, 181-209.

[F] H. Furstenberg, Boundary theory and stochastic processes on homogeneous spaces. Harmonic analysis on homogeneous spaces (Proc. Sympos. Pure Math., Vol. XXVI), 193-229. Amer. Math. Soc., Providence, R.I., 1973.

[GO] A. Gorodnik and H. Oh, Orbits of discrete subgroups on a symmetric space and the Furstenberg boundary. In preparation.

[Ka] D. A. Každan, Uniform distribution on a plane, Trudy Moskov. Mat. Obšč. 14, 299-305, 1965.

[Kn] G. Knieper, On the asymptotic geometry of nonpositively curved manifolds. Geom. Funct. Anal. 7 (1997), no. 4, 755-782.

[L] F. Ledrappier, Distribution des orbites des réseaux sur le plan réel. C.R. Acad. Sci. Paris Sr. I Math. 329, no. 1, 61-64, 1999.

[Ma] F. Maucourant, Approximation diophantienne, dynamique des chambres de Weyl et répartitions d'orbites de réseaux. $\mathrm{PhD}$ Thesis, Université de Lille, 2002.

[Mo] G. D. Mostow, Strong rigidity of locally symmetric spaces. Annals of Mathematics Studies, No. 78. Princeton University Press, 1973.

$[\mathrm{N}] \quad$ A. Nogueira, Orbit distribution on $\mathbb{R}^{2}$ under the natural action of $\mathrm{SL}(2, \mathbb{Z})$. Indag. Math. (N.S.) 13 (2002), no. 1, 103-124.

[R] T. Roblin, Ergodicité et équidistribution en courbure négative. Mémoires de la SMF 95 (2003).

[S] N. Shah, Limit distribution of expanding translates of certain orbits on homogeneous spaces. Proc. Indian Acad. Sci. Math. Sci. 106 (1996), no. 2, 105-125.

[T] J. Tits, Représentations linéaires irréductibles d'un groupe réductif sur un corps quelconque. J. Reine Angew. Math. 247 (1971), 196-220.

[W] G. Warner, Harmonic analysis on semi-simple Lie groups. I. Die Grundlehren der mathematischen Wissenschaften, Band 188. Springer-Verlag, New York-Heidelberg, 1972.

Mathematics Department, University of Michigan, Ann Arbor, Mi 48109 USA

E-mail address: gorodnik@umich.edu

École normale supérieure de Lyon, Unité de Mathématiques Pures et Appliquées, UMR CNRS 5669, 46, allée D'Italie, 69364 Lyon Cedex 07 France

E-mail address: Francois. MAUCOURANT@umpa.ens-lyon.fr 